\title{
RECHERCHE DES DÉBITS ADMISSIBLES DANS LES CANALISATIONS PAR LE CALCUL DES PROBABILITÉS
}

\section{DETERMINATION OF PIPES SUPPLYING AND EMPTYING NUMEROUS INTERMITTENTLY AND INDEPENDENTLY USED APPARATUSES}

par R. BOLANT

Ingénieur I. E. G., Licencié ès Sciences

English synopsis p. 228

\section{INTRODUCTION}

Le calcul des canalisations devant assurer l'alimentation ou la vidange d'un ensemble complexe d'appareils a déjà fait l'objet d'assez nombreux articles, mais il n'apparaît pas que cette question ait encore reçu une solution vraiment satisfaisante.

En effet, un tel calcul peut se heurter à deux sortes de difficultés:

Dans le cas où les canalisations alimentent des appareils à fonctionnement continu, ou bien, quand un cahier des charges impose le débit que devra pouvoir assurer chaque conduite, le calcul se limite en général à une estimation de pertes de charge. Les difficultés rencontrées concernent le choix des hypothèses et des formules employées, choix qui doit être judicieux, mais sur lequel nous n'envisageons pas de nous étendre ici, la littérature s'y rapportant étant, d'ailleurs, abondante.

Au contraire, dans une installation comprenant un certain nombre d'appareils de puisage ou de vidange à fonctionnement intermittent, i! ne saurait être question, économiquement, de dimensionner chaque canalisation pour le débit maximum qu'elle devrait fournir si tous les appareils étaient à même d'être normalement et simultanément en usage; en l'absence d'indications fournies par un cahier des charges (indications plus ou moins arbitraires d'ailleurs) le problème se pose donc très souvent de déterminer quel est le débit minimum susceptible d'être assigné à chaque canalisation, de manière à ce que l'ensemble présente encore un fonctionnement satisfaisant.

C'est la recherche de ce compromis le plus favorable entre les conditions de prix de revient et d'encombrement, d'une part, et le fait que chaque appareil doit pouvoir assurer le service qu'on en attend, d'autre part, que nous nous proposons de traiter.

De l'avis de techniciens autorisés, trop peu d'ouvrages envisagent ce second problème, et ne le font que d'une manière trop simpliste.

A priori, on peut penser, en effet, dans la majeure partie des cas, que cette détermination repose sur des éléments trop variés, mal définis et aléatoires, pour qu'une solution vraiment rationnelle puisse intervenir; au cours d'une journée, le débit d'une conduite pourra être fonction :

- de l'instant considéré,

- du nombre des appareils en état de fonctionnement,

- de leur nature.

- de leur disposition,

- des différents besoins et desiderata des utilisateurs (débits et volumes demandés susceptibles de variations notables, même pour des appareils de types semblables),

- enfin, de l'ensemble des canalisations, de la conduite d'amenée et de la pression à l'entrée.

Aussi, a-t-on généralement recours en pratique, à une règle de l'art assez mal définie, en s'appuyant sur l'expérience d'installations en service.

II s'ensuit que, si le fonctionnement est à peu près correct dans les cas les plus courants - la tendance est de calculer «large», ainsi que nous le verrons plus loin - dans la plupart des installations particulières la détermination se révèle plus hasardeuse encore et il n'est pas rare 
de constater, par la suite, que le dimensionnement est très exagéré, ou, ce qui est pius grave, que le fonctionnement s'avère défectueux.

Le présent article se propose donc d'indiquer la voie dans laquelle un calcul valable peut être entrepris. Cette détermination des débits admissibles s'appuiera sur le calcul des probabilités, qui, seul, apparait comme pouvant apporter une solution, sinon très simple, du moins correcte.

A notre connaissance, une tentative a déjà été faite en ce sens; malheureusement, des simplifications illégitimes, l'emploi abusif de formules asymptotiques en dehors de leur domaine de validité ne permettent pas de considérer que la question a été, pour autant, résolue.

Nous nous proposons, tout d'abord, de préciser certaines notions - telles que ce qu'on appelle généralement "fonctionnement correct» - ce qui nous amènera à dégager la définition de quelques quantités propres à intervenir dans une détermination rationnelle et à indiquer des ordres de grandeur de ces quantités. Ensuite, nous rappellerons très succinctement quelques propositions fondamentales du calcul des probabilités.

Nous appliquerons les résultats acquis à la recherche considérée dans des cas d'installations de plus en plus complexes.

Chemin faisant, nous soulignerons les résultats principaux susceptibles d'intérêt général et ferons des rapprochements avec des installations existantes.

\section{DEFINITION DES GRANDEURS UTILISÉES}

\section{Qualité de fonctionnement d'une installation.}

Demander que le débit nominal de chaque appareil puisse être assuré à tout moment revient à exiger que l'installation soit calculée pour le débit maximum correspondant au plus grand nombre possible d'appareils pouvant se trouver en fonctionnement simultané à un certain moment. Economiquement, pour des appareils dont les fonctionnements sont indépendants les uns des autres et qui ne sont chacun en activité que pendant une faible fraction du temps, ce point de vue est une hérésie.

Il faudra, par conséquent, admettre qu'un appareil ne fournisse pas tout le temps le débit qu'on lui demande; mais, par ailleurs, il sera nécessaire, pour qu'une installation réponde de façon satisfaisante aux besoins de tous les utilisateurs, que chaque appareil ne soit pas empêché de conner son débit nominal pendant plus d'une faible fraction de son temps d'utilisation.

II semble donc logique d'admettre que l'installation est d'autant plus parfaite qu'elle est susceptible d'assurer, sur chaque appareil, le débit nominal pendant une plus grande fraction du temps d'utilisation.

Nous choisirons pour définir précisément la qualité de fonctionnement, la plus faible des valeurs que prend la probabilité d'obtenir de cet appareil, au cours de la période d'utilisation, un débit au moins égal au débit nominal.

De façon concrète, dire que la probabilité de fonctionnement correct est $m$ signifie que, sur un grand nombre d'observations portant sur la même certaine période du temps d'utilisation le rapport :

lemps de fonctionnement correct (débit $\geqslant$ débit nominal) temps de fonctionnement total

tend vers $m$ pour l'appareil considéré. II convient de remarquer dès à présent que le fait de calculer une installation pour qu'un certain appareil réponde à la condition précédente ne peut empêcher que, lors d'un fonctionnement isolé, le rapport ci-dessus relatif à ce seul fonctionnement soit très faible ou nul. II n'y a là aucune contradiction, car nulle garantie ne peut être donnée quant à un fonctionnement unique si l'installation n'a pas été calculée pour assurer le maximum de débit possible.

D'autres définitions peuvent évidemment être imaginées relativement à la condition de qualité ; par exemple, on pourrait convenir de prendre l'une des deux définitions suivantes:

- la qualité de fonctionnement d'un appareil donné sera déterminée par la plus faible des valeurs que prend la probabilité d'obtenir sur cet appareil, au cours de sa période d'utilisation, un débit supérieur à une certaine fraction de son débit nominal,

ou bien :

- la qualité de fonctionnement d'un appareil donné sera fournie par la plus faible des valeurs prise par la probabilité que ne s'écoulent plus de $t$ secondes sans que l'appareil ne puisse assurer au moins une certaine fraction déterminée de son débit nominal.

Ces conditions ne sont pas strictement équivalentes, et on peut considérer, par exemple. que la dernière constitue un critère mieux adapté aux exigences de la pratique que les deux 
précédentes. Néanmoins, nous adopterons la première en raison de sa simplicité.

En effet, il est difficile de faire intervenir, au sujet des deux autres définitions, des hypothèses qui soient indépendantes des pertes de charge intéressant les différents appareils, pertes de charge elles-mêmes fonctions du dimensionnement de l'installation que l'on calcule. D'autre part, les hypothèses que nous devrons obligatow rement faire au sujet des debits et des temps de fonctionnement ne justifieraient pas des complications considérables de calcul destinées à augu menter quelque peu la valeur thérique de la détermination. Dans la suite, nous serons d'all leurs amenés à envisager séparément, dans toute la mesure du possible, les questions de probabi lité de fonctionnement des différents appareils, d'une part, et les considérations de pertes de charge qui leur sont attachées, d'autre part. $C e$ point de vue apparaît légitime en ce sens que le calcul que nous avons en vue ne peut se flatter d'une plus grande précision que celle que l'on peut attendre de la détermination des données, laquelle est sujette, en général, à quelque incertitude.

La valeur $m$ de la probabilité de fonctionnement - relative à la première définition devra être adaptée aux exigences diverses que pourront légitimement manifester les utilisateurs; un lavage chimique par exemple, pourra être plus ou moins délicat et, suivant les cas. exiger une grande qualité (peut être $m=0.98$ ) ou permettre un fonctionnement moins parfait $(\mathrm{m}=0,90)$.

De même, en ce qui concenne les installations sanitaires, on s'attachera moins à la régularite du débit alimentant une baignoire qu'a celle du robinet qui se trouve sur l'évier.

En conséquence, la qualité d'ensemble d'une installation sera jugée acceptable quand chacun des appareils constituant cette installation aura au moins pour qualité propre celle qu'on lui a assignée.

\section{Période de pointe.}

Une installation quelconque n'est pas soumise, dans la majorité des cas, au même régime de fonctionnement à toutes les heures dé la journée; au contraire, il existe le plus souvent une certaine période pendant laquelle l'ensemble des utilisateurs fait preuve d'une activité plus grande et sensiblement constante en moyenne.
C'est ce que confirmeraient les statistiques relatives aux besoins de tous les utilisateurs pendant un très grand nombre de jours, ou même des hypothèses raisonnables sur l'activité de ces utilisateurs.

C'est le laps de temps, précédemment défini, qu'il convient de retenir pour effectuer un calcul correct de l'installation, puisque c'est alors que la demande est la plus forte et que la qualité de fonctionnement risque d'atteindre son minimum. Nous appellerons dans la suite cette période, période de pointe $T$.

La détermination de cette période de pointe sera plus ou moins délicate et susceptible, suivant les cas, d'une précision variable. II importe de noter à ce sujet, que la statistique à laquelle nous avons fat allusion ci-dessus est relative aux bosoins reels et non à ceux auxquels peut repondre une installation existante qui ne donne peutetrepas enterement satistaction

A the dexemple, la pertode de pointe pour une installation industrielle dappareils indépendants, à fonctionnement intermittent, destinés à fournir toujours le meme travail, s'étendra sur l'ensemole des heures de présence du personnel.

Par contre, sl une ushe comporte une installation de douches les usagers étant admis dans les cabines simultanement, la période de pointe n'excedera par les quelques minutes pendant lesquelles 16 presque totalité des robinets sont en activito

Manifostmonent, il n'y a pas intérêt à appliquer la presente btude à une telle installation qui doit ctresensiblement calculée pour le maximum de debit possible.

Une dotermination pourra être plus délicate par le fait que tous les appareils utilisés présentent eux mêmes des pointes à des instants differents de la journée. Nous en verrons un exemple concret plus loin.

\section{Volume moyen nécessaire.}

Pendant la période de pointe, chaque utilisareur aura besoin d'obtenir ou d'évacuer un certain volume de fluide. Pour savoir quel volume est nécessaire, considérons un grand nombre d'utilisateurs et chacun d'eux pendant un certain nombre de jours; nous constaterons que. pour obtenir un même résultat, procéder à un certain lavage ou à une opération chimique donnée, nettoyer un ensemble d'objets, etc..., le 
volume demandé chaque fois sera, dans l'ensemble, peu différent du volume $V$ tel que:

$$
V=\frac{\text { Volume total demandé }}{\text { Nombre total d'opérations }}
$$

Nous pourrons dès lors admettre que $V$ est le volume nécessaire à l'obtention du résultat recherché ; nous le définirons comme le volume moyen nécessaire.

En général, une opération industrielle demandera un volume de fluide assez bien défini et relativement facile à déterminer.

\section{Débił nominal d'un appareil.}

Deux considérations peuvent intervenir dans le choix du débit à introduire dans les calculs:

D'une part, il est possible;-en procédant comme précédemment, de définir le débit moyen d'un appareil ayant une activité toujours sensiblement la même, grâce à la formule:

$$
m=\frac{\text { Volume total débité par un appareil }}{\text { Temps total de fonctionnement }}
$$

D'autre part, il semble logique d'admettre que tout utilisateur est en droit d'obtenir le débit pour lequel l'appareil est construit, c'est-à-dire le débit nominal.

Dans la majeure partie des cas, pour une installation qui n'a pas été trop largement calculée, ces deux débits sont voisins et nous adopterons, en conséquence, le débit nominal $Q$.

La détermination est ici encore plus simple dans le cas des usages industriels que dans celui des installations sanitaires.

\section{Temps moyen de fonctionnement.}

Nous prendrons pour temps moyen de fonctionnement d'un appareil donné pendant la période de pointe de l'installation, dont il fait partie, le quotient :

$$
d=\frac{\text { Volume moyen nécessaire pendant la pointe }}{\text { Débit nominal de l'appareil }}
$$

La détermination de ce temps repose donc sur les hypothèses que nous avons précédemment faites ; il est en particulier possible que la valeur trouvée ainsi soit un peu faible si l'utilisateur n'a pas toujours demandé à l'appareil son débit nominal. Dans ce cas, on peut prendre pour temps de fonctionnement une valeur se rapprochant de la valeur réelle, ce qui revient à introduire un volume moyen un peu plus grand.
D'autre part, un appareil ne pouvant fournir son débit nominal $Q$ qu'avec la probabilité $m$, le volume de fluide effectivement obtenu pendant le temps de fonctionnement d fluctuera au-dessous de la valeur : Qd...

Comme précédemment, une légère correction peut être effectuée, qui ne justifie d'ailleurs pas quand la qualité de fonctionnement est suffisante, ce qui est le cas général.

En résumé, nous remplacerons, pendant la période de pointe à laquelle se trouve soumise l'installation, les utilisateurs habituels par des opérateurs agissant indépendamment les uns des autres et exigeant de chaque appareil, pendant la période considérée :

- un débit constamment égal au débit nominal,

- un temps de fonctionnement toujours identique et égal au temps de fonctionnement moyen.

Après avoir rappelé quelques propositions relatives au calcul des probabilités, nous appliquerons très simplement les définitions que nous venons de donner à la détermination que nous avons en vue.

\section{RAPPEL DE QUELQUES NOTIONS FONDAMENTALES DU CALCUL DES PROBABILITÉS}

\section{Probabilité continue.}

Comme on le sait, la probabilité de réalisation d'une certaine éventualité est le rapport du nombre des cas favorables à cette éventualité au nombre des cas possibles, tous les cas étant regardés comme également susceptibles de se produire.

Si nous désignons par p la probabilité d'un certain événement, celle de la réalisation de l'événement contraire q sera :

$$
q=1-p
$$

d'après la précédente définition, une certitude correspondant à une probabilité égale à 1 .

Lorsque le nombre des cas possibles n'est pas fini, il est nécessaire de faire intervenir une «probabilité continue» qui étend la définition donnée ci-dessus; on peut concevoir cette probabilité continue comme la limite du rapport des cas favorables aux cas possibles quand le nombre des cas augmente indéfiniment. 
Considérons, par exemple, un point $M$ pris au hasard sur un segment de droite $A B$ et cherchons quelle est la possibilité pour qu'il soit compris entre les points $C$ et $D$ donnés sur ce segment. Nous pouvons, dans ce but, découper le segment $A B$ en $N$ parties égales et, considérant qu'il n'y a pas de raison que le point $M$ appartienne plus à l'un qu'à l'autre des petits segments élémen. taires, donner à la probabilité cherchée la valeur:

Nombre des segments etomentaires intéreurs a $\mathrm{CD}$

$$
\text { N }
$$

En faisant tendre $N$ vers l'infini de quelque manière qu'on désire, la limite de ce rapport est : $\frac{C D}{A B}$. Notons, puisque nous en aurons besoin tout-à-l'heure, que cette probabilité ne se trouverait pas modifiée par le fait que le segment $C D$ serait brisé en plüsieurs segments plus petits disposés suivant $\mathrm{OA}$, de façon quelconque, mais sans recouvrement.

\section{PROBABILITÉS TOTALES ET PROBABILITÉS COMPOSÉES}

\section{Principe des probabilités composées.}

Si un événement suppose la réalisation de deux événements $A$ et $B$, sa probabilité est le produit de la probabilité de A par la probabilité pour que $B$ se produise, sachant que $A$ s'est produit.

En effet, les éventualités à considérer dans ce cas sont les suivantes:

- il se produit $A$ et B; cette éventualité correspondant à $n_{1}$ cas possibles,

- il se produit $A$ et non $B ; n_{2}$ cas possibles,

- il se produit $B$ et non $A ; n_{3}$ cas possibles,

- il ne se produit ni $A$ ni $B ; n_{4}$ cas possibles. Soit en tout : $N$ cas.

Par définition, probabilité de l'événement $A B$ :

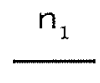

$N$

Or, la probabilité de $A$ est $: \frac{n_{1}+n_{2}}{N}$

et celle de $B$, sachant que A s'est déjà produit :

$$
\frac{n_{1}}{n_{1}+n_{2}}
$$

L'énoncé ci-dessus en résulte; il s'étend de proche en proche à des événements demandant l'intervention de facteurs de plus en plus nombreux. Il va de soi que la restriction de réalisa. tion préalable des événements déjà envisagés n'a plus de raison d'être quand ces événements sont indépendants les uns des autres. En particulier, la probabilité de réalisation $\mathrm{P}$ d'un état demandant l'existence simultanée de $n$ facteurs indépendants ayant eux-mêmes les probabilités $p_{1}$, $\mathrm{P}_{2} \ldots \mathrm{P}_{n}$. de se produire, sera :

$$
P=p_{1} \cdot p_{2} \ldots p_{n} \text {. }
$$

\section{Principe des probabilités totales.}

Si un événement peut se produire de plusieurs manières s'excluant mutuellement, la probabilité de cet événement est la somme des probabilités partielles correspondant aux diverses manières.

$\mathrm{Si}$ les probabilités partielles correspondant respectivement à $n_{1}$ cas favorables, $n_{2}$ cas favorables ... s'excluant mutuellement sur $N$ cas possibles, la réalisation de l'événement considéré, par un moyen quelconque, a pour probabilité :

$$
p=\frac{n_{1}+n_{2}+\ldots}{N}
$$

ce qui démontre cette propriété bien intuitive.

Ajoutons que si les cas favorables ne sont pas distincts il y a moins de cas favorables que: $n_{1}+n_{2} \ldots$ et la probabilité est plus faible que la somme des probabilités partielles.

\section{Valeur la plus probable.}

Lorsqu'une grandeur peut prendre des valeurs diverses, on appelle valeur la plus probable celle qui correspond à la probabilité de réalisation maximum.

Ces quelques notions fort simples étant rappelées, revenons au problème posé.

\section{CALCUL DES PROBABILITÉS DE FONCTIONNEMENT}

\section{Probabilité de fonctionnement d'un appareil isolé.}

Soit, figurée suivant un axe Ot, une certaine période $T$ comprise entre les instants représentés par les points $A$ et $B$ et supposons qu'un certain appareil ait fonctionné pendant cette 
période, entre les instants $C$ et $D$, puis $E$ et $F$, etc... en tout, pendant une durée $d$.

II résulte alors de la définition donnée plus haut, relative à la probabilité continue, que la probabilité de fonctionnement de l'appareil considéré, à un instant quelconque de la période $T$, est égale à $\frac{d}{T}$.

Or, comme on vient de le voir, cette probabilité qui intervient dans la définition adoptée de la qualité d'une installation, est indépendante de la manière dont l'utilisateur fait usage de l'appareil, pour autant que la durée de fonctionnement d soit la même.

Ainsi, n'a-t-on aucune hypothèse à formuler quant à la distribution et à la durée des fonctionnements pendant la période de pointe.

\section{Probabilité de fonctionnement simultrané de $k$} appareils parmi n appareils semblables.

L'étude des «épreuves répétées » du Calcul des Probabilités s'applique à cette détermination.

La probabilité de fonctionnement de $k$ appareils donnés est, d'après le principe des probabilités composées, égale à : $p^{k}$

La probabilité de non-fonctionnement des (n-k) appareils restants est, de même:

$$
(1-p)^{n-k}=q^{n-k}
$$

La probabilité de fonctionnement simultané de $k$ appareils déferminés parmi les $n$ appareils existants est, par conséquent :

$$
p^{k} q^{n-k}
$$

Comme nous ne nous attachons pas au fonctionnement de $k$ appareils donnés, mais de $k$ appareils quelconques, il convient de multiplier la précédente probabilité par le nombre de manières différentes dont il est possible de choisir $k$ appareils parmi $n$ (principe des probabilités totales). La probabilité cherchée est donc:

$$
P_{k}=C_{n}^{k} p^{k} q^{n-k}=\frac{n !}{k !(n-k) !} p^{k} q^{n-k}
$$

C'est le terme de rang $(k+1)$ du développement de:

$$
(p+q)^{n}
$$

Cette formule permet de dresser un tableau de la variation de la probabilité en fonction du nombre $k$ d'appareils en fonctionnement simultané. Si le nombre total d'appareils $n$ est assez grand, et si la probabilité de fonctionnement d'un appareil isolé $\mathrm{p}$ est faible, on vérifie que la probabilité correspondant au fonctionnement simultané d'une importante fraction de l'installation est extrêmement petite.

$1 /$ peut être intéressant de rechercher quel est le débit le plus probable de la précédente installation, soit hq.

La condition déterminant $h$ est que la double inégalité

$$
P_{h-1} \leqslant P_{h} \geqslant P_{h+1}
$$

soit satisfaite. Or,

$$
\begin{aligned}
& \frac{P_{h}}{P_{h-1}}=\frac{n-h+1}{h} \cdot \frac{p}{q} \\
& \text { et } \frac{P_{h}}{P_{h+1}}=\frac{h+1}{n-h} \frac{q}{p}
\end{aligned}
$$

et, en se souvenant que:

$$
\begin{aligned}
& p+q=1, \text { il vient : } \\
& n p-q \leqslant h \leqslant(n+1) p
\end{aligned}
$$

d'où le résultat: pour un nombre $n$ d'appareils déterminé, le nombre le plus probable d'appareils en fonctionnement simultané est $h$ entier, tel que :

$$
p-\frac{q}{n} \leqslant \frac{h}{n} \leqslant p+\frac{p}{n}
$$

Soulignons bien le fait que ce résultat, qui peut être très rapidement atteint, ne suffit pas à déterminer le débit qu'il faut assurer à l'installation.

\section{Généralisation.}

Supposons que nous envisagions un ensemble de : $k$, appareils ayant une probabilité individuelle de fonctionnement $p_{1}$ et un débit $Q_{1}, k_{2}$ appareils ayant une probabilité $p_{2}$ et un débit $Q_{2}$, etc...

Plaçons-nous encore dans le cas où l'installation permet l'obtention désirée de tous les débits nominaux sur tous les appareils. Chaque débit possible de l'ensemble considéré sera de la forme:

$$
\bar{Q}=\Sigma \alpha_{i} \cdot Q_{i} \quad \text { avec }: 0 \leqslant \alpha_{i} \leqslant k_{i}
$$

Certains de ces débits $\bar{Q}$ pourront être obtenus de plusieurs manières différentes s'il existe des débits $Q_{i}$ entre eux dans un rapport suffisamment simple. 
Soit un débit $\bar{Q}$ possible de $m$ façons: pour un certain mode de réalisation, sa probabilité $P_{\bar{Q}}$ sera exprimée par le produit :

$$
\mathrm{P}_{\overline{\mathrm{Q}}}=\pi \cdot \mathrm{p}_{\mathrm{i}}^{\alpha_{i}} \cdot \mathrm{q}_{\mathrm{i}} \mathrm{p}_{\mathrm{i}}(\beta=\mathrm{k}-\alpha)
$$

(les indices i désignant des appareils en activité et les indices $j$ des appareils au repos).

Pour un second mode, la probabilité sera $P^{\prime} \bar{Q}$ etc..., en vertu du principe des probabilités composées.

Pour l'ensemble de tous les modes d'obtenir le débit Q, la probabilité résultante sera la somme des probabilités partielles:

$$
\overline{\mathrm{P}}=\Sigma \cdot \mathrm{P}_{\overline{\mathrm{Q}}}
$$

\section{Probabilité de fonctionnement normal d'un appareil.}

Supposons réalisée une installation comprenant un certain nombre d'appareils montés sur une colonne d'alimentation ou de vidange unique et répondant aux deux conditions suivantes :

- d'une part, le débir maximum que peut assurer la canalisation principale est $\mathrm{M} \mathrm{l} / \mathrm{s}$.;

- d'autre part, le débit de n'importe lequel des appareills demeure égal, quand il fonctionne, à son débit nominal, pour autant qu'à l'instant considéré, la somme des débits nominaux demandés par l'ensemble de rous les appareils en service n'excède pas $M$.

Ainsi, la condition nécessaire et suffisante pour qu'à un instant donné le fonctionnement normal d'un appareil déteminé soit possible. c'est-à-dire qu'il puisse foumit son débit nomi nal $Q$, est que la somme des débits demandés au même instant par l'ensemble des autres appa. reils soit inférieure à $M-Q_{1}$.

Ceci va nous permettre de calculer la proba bilité qu'un appareil a de fonctionner normalew ment dans les conditions données et de déter. miner par conséquent quelle est la qualité de l'installation en ce qui concerne l'appareil considéré.

Replaçons-nous d'abord dans le cas d'une installation de $n$ appareils identiques ayant un débit moyen Q. Un appareil donné pourra fonctionner normalement si, le débit de la canalisation principale pouvant assurer $M=x Q$, il n'y a pas, au même instant, plus de $(x-1)$ autres appareils en fonctionnement.

Or. la probabilité d'une telle éventualité est:

$$
\bar{P}_{x}=P_{0}+\ldots .+P_{x-1}
$$

somme des probabilités correspondant à des débits $O, Q, 2 Q \ldots(x-1) Q$, pour les $n-1$ appareils restants.

\section{Généralisation.}

Soient maintenant des appareils demandant des débits moyens différents et ayant des probabilités individuelles également différentes; la qualité de fonctionnement d'un appareil donné de debit $Q_{i}$ se détermine de façon analogue en considérant la somme des probabilités $P$ pour tous les débits possibles inférieurs ou égaux à $M-Q_{i}$ que peuvent demander les appareils restants.

Il va de soi que si les appareils sont différents, la qualité de fonctionnement attachée à chacun d'eux peut fort bien être très variable avec l'appareil considéré.

\section{DÉBIT A ADMETTRE POUR UNE CANALISATION}

II faut tout d'abord choisir les débits convenables et fixer le temps de fonctionnement de chaque appareil en accord avec l'usage qu'on en attend pendant la période de pointe.

\section{Cas de $\mathbf{n}$ appareils semblables.}

Les appareils seront dits semblables quand leurs débirs nominaux et leurs probabilités individuelles de fonctionnement seront les mêmes.

Soit m la valeur minimum qu'on est disposé à admetre pour la qualité de fonctionnement de limstallation en étude.

La qualité de fonctionnement sera donc jugée suffisante quand la probabilité d'obtenir à un instant quelconque de la période de pointe le débit nominal sur un appareil sera au moins égale à $\mathrm{m}$. Or, cette probabilité vient d'être calculée pour un débit $\times Q$ de la canalisation; il suffira, par conséquent, de développer la somme $P_{1}+P_{1}+\ldots$ jusqu'à ce qu'elle soit égale ou supérieure à la valeur $\mathrm{m}$ recherchée.

Le débit à admettre, bien entendu égal à un multiple du débit unitaire nominal, sera égal à $\times Q$ si le dernier terme introduit dans la somme est relatif à un débit que nous posons égal à (x-1) $Q$.

Pour répondre aux conditions que nous nous sommes fixées, le dimensionnement devra être tel que le débit nominal soit assuré quels que 
soient les appareils en service si le nombre de ceux-ci n'excède pas $x$; le calcul s'effectuera donc en supposant que les appareils qui demandent le débit total $\times Q$ sont ceux auxquels sont attachées les plus fortes pertes de charge par exemple les plus éloignées ou ceux dont les cotes sont les plus élevées.

La qualité de fonctionnement des appareils ayant les plus petites pertes de charge pourra être bien meilleure que le minimum admissible au voisinage duquel travaillent les appareils les moins favorisés, mais ils ne devront pas pouvoir débiter plus que le débit $Q$, sinon l'hypothèse de $n$ appareils semblables n'est pas valable et on est dans le cas général.

\section{Cas de $\mathbf{n}$ appareils quelconques.}

Soit $m_{i}$ la valeur minimum qu'on peut admettre quant à la qualité de fonctionnement de l'appareil $A_{i}$; nous appellerons comme précédemment $Q_{i}$ le débit et $p_{i}$ la probabilité de fonctionnement de cet appareil considéré isolément.

Nous envisagerons les débits $\bar{Q}$ croissants que l'ensemble de tous les appareils montés sur la colonne en étude est susceptible de demander, et nous calculerons la probabilité de réalisation de chacun de ces débits. Nous serons ainsi en mesure de dresser un tableau donnant le régime de fonctionnement de la canalisation.

La qualité relative à l'appareil $A_{i}$ sera au moins égale à la somme des probabilités pour que tous les appareils, sauf $A_{i}$, demandent des débits inférieurs à $M-Q_{i}$ si l'installation est dimensionnée pour assurer un débit $M$ dans les conditions les plus défavorables. II conviendra donc de choisir $M$ - égal à l'un des débits que peut demander l'ensemble de l'installation - pour que la somme des probabilités précédentes surpasse la qualité $m_{i}$ requise pour l'appareil $A_{i}$.

Pratiquement, une disposition assez convenable semble être la suivante (fig. 1).

Nous supposerons, pour commencer, que les débits sont tous différents. Nous les rangerons par valeurs croissantes et les écrirons sur une ligne; nous disposerons en dessous les probabilités $p_{i}$ correspondantes, puis les probabilités complémentaires $q_{i}$.

Nous pourrons ensuite dresser facilement un tableau où se trouvent alignés :

- en haut, les premiers débits $\bar{Q}$ croissants que peut demander l'ensemble de l'installation,

\begin{tabular}{|c|c|c|c|c|c|}
\hline Débits & $Q_{1}$ & $Q_{2}$ & $Q_{3}$ & & \\
\hline Appareils & $A_{1}$ & $\mathrm{~A}_{2}$ & $A_{3}$ & & \\
\hline Probabilites $p$ & $p_{1}$ & $P_{2}$ & $P_{3}$ & & \\
\hline Probabilités $q$ & $q_{1}$ & $q_{2}$ & $a_{3}$ & & \\
\hline \multicolumn{6}{|c|}{ Tableau $T_{1}$} \\
\hline Débits & $\bar{Q}_{1}$ & $\bar{Q}_{2}$ & $\bar{Q}_{3}$ & $\bar{Q}_{4}$ & \\
\hline Appareils & $A_{1}$ & $A_{2}$ & $A_{1}+A_{2}$ & $A_{3}$ & \\
\hline Probabilités $\bar{P}$ & $\bar{P}_{1}$ & $\bar{P}_{2}$ & $\bar{P}_{3}$ & $\bar{P}_{4}$ & \\
\hline
\end{tabular}

- en dessous, les probabilités correspondantes que nous avons vu comment calculer.

Ensuite, en prenant pour valeur admissible dans la canalisation l'un des débits $\bar{Q}$ semblant, à priori, convenable, nous chercherons à vérifier que la qualité de fonctionnement de l'appareil $A_{i}$ est égale ou supérieure à $m_{i}$.

Cette vérification est rapide : il nous suffit de relever dans le tableau précédent toutes les probabilités relatives aux débits où n'intervient pas l'appareil $A_{i}$, en nous limitant aux débits inférieurs ou égaux à $M-Q_{i}$. La sommeo $i$ de ces probabilités, divisée par $q_{i}$, fournit la qualité de fonctionnement cherchée.

Très peu d'essais conduisent à la valeur $M$ qui est le débit minimum assurant la qualité désirée sur chaque appareil ; $M$ est le débit qu'il convient d'admettre pour le calcul de la canalisation.

Le dimensionnement de la colonne sera effectué en supposant que la combinaison des appareils demandant le débit $M$ retenu est celle qui correspond aux plus grandes pertes de charge possibles. Dans certains cas, quand les combinaisons susceptibles de demander le débit $M$ correspondront à des appareils favorisés au point de vue pertes de charge, il faudra vérifier que la canalisation assure effectivement les débits peu inférieurs à $M$ pouvant être demandés par des appareils plus défavorisés.

Pratiquement, la méthode qui précède est d'une application simple, et le lecteur s'en convaincra en se reportant, dès maintenant, à quelques exemples concrets de détermination fournis plus loin. 
Lorsqu'un débit $\bar{Q}$ peut être obtenu de plusieurs manières différentes, il suffit de séparer en autant de colonnes les probabilités $P$ correspondantes pour mener à bien le calcul exactement comme ci-dessus.

$\mathrm{Si}$ nous supposons maintenant qu'il existe dans l'installation $\mathbf{n}$ appareils $A_{i}$ semblables, nous pourrons, en vue d'alléger l'écriture, ne réserver qu'une seule colonne pour un débit $\overline{\mathrm{Q}}$ de l'installation supposant l'activité simultanée de $\mathrm{k}$ quelconques des $n$ appareils $A_{i}$.

Mais il faudra alors, au moment de l'évaluation de la qualité de fonctionnement du groupe $A_{i}$, faire comme ci-dessus, abstraction de l'un des appareils $A_{i}$. ce qui revient ici à multiplier la probabilité (d'obtenir $\bar{Q}$ quels que soient les $k$ appareils $A_{i}$ en service) par le facteur $\frac{n-k}{n}$. n

En effet, les appareils $A_{\text {i }}$ interviennent dans le produit donnant $P$, par le facteur :

$$
C_{n}{ }^{k} \quad D_{i}{ }^{k} \quad q_{i}{ }^{n-k}
$$

Or. il faut faire en sorte que ce terme soit remplacé par :

$$
c_{(n-1)}^{k} p_{i}^{k} q_{i}^{(n-k-1)} \times q_{i}
$$

dans le calcul de la somme $r_{i}$. Il est donc nécessaire de multiplier la première expression par:

$$
\frac{c_{(n-1)}^{k}}{c_{n}^{k}}=\frac{n-k}{n}
$$

\section{Cas des canalisations principales.}

En ce qui concerne cette question, nous nous limiterons ici au cas d'une canalisation principale (ou collecteur) sur laquelle se trouvent montées des canalisations identiques intéressant le même ensemble d'appareils, semblables ou non (fig. 2).

La détermination de la période de pointe, des probabilités élémentaires de fonctionnement et des débits nominaux demeurera la même.

Avant de nous attaquer à la recherche proprement dite :

- du débit admissible pour les canalisations secondaires: $M_{1}$,

- du débit admissible pour la canalisation principale $\mathrm{M}_{2}$,

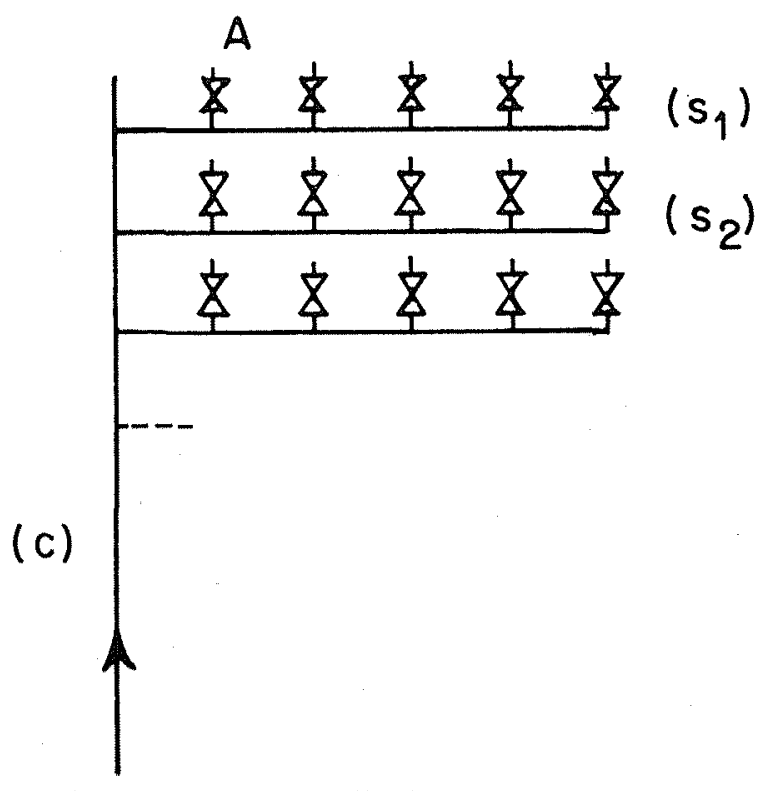

Fig. 2

Pour une certaine installation, nous supposerons cette installation donnée, et calculerons la qualité de fonctionnement d'un appareil $A_{i}$.

D'une part, si le collecteur n'intervenait pas pour limiter parfois le débit demandé par une canalisation secondaire, la qualité de l'appareil $A_{i}$ se déterminerait comme précédemment grâce à un tableau T, (fig. 1).

D'autre part, connaissant le débit maximum $M_{1}$ des canalisations secondaires et les caractéristiques de tous les appareils, nous serions théoriquement à même d'établir un tableau $T_{2}$ (fig. 3) fournissant les probabilités des débits que peuvent demander l'ensemble des appareils, à l'exclusion de ceux montés sur la même canalisation que l'appareil $A_{i}$ considéré.

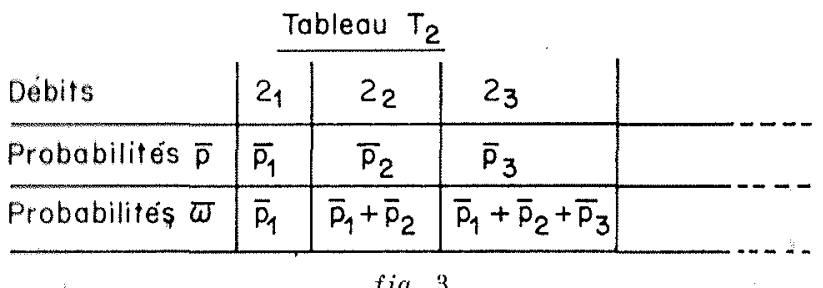

fig. 3

En nous aidant de ces deux tableaux, nous déterminerions la qualité cherchée.

En effet, d'après $T_{1}$, soit $\bar{P}$ la probabilité d'un débit $\bar{Q}$ auquel participe $A_{i}$ dans la canalisation secondaire considérée. 
D'après $T_{2}$, soit (1) la probabilité que le débit demandé par l'ensemble des autres canalisations secondaires soit inférieur à $M_{2}-\bar{Q}$.

Le débit normal sera obtenu dans ce cas avec la probabilité :

$$
\mathrm{P}_{(1)}^{-}
$$

La somme de toutes ces probabilités correspondant à tous les débits $\bar{Q}$ où participe $A_{i}$ fournit la probabilité cherchée pour cet appareil.

La recherche du dimensionnement du collecteur et des canalisations secondaires devra tenir le plus grand compte des longueurs relatives et des données particulières du cas considéré en vue d'obtenir le compromis le meilleur. Par exemple, si le collecteur est relativement très long, il y aura intérêt à réduire au maximum le débit qui lui correspond, quittè à devoir, pour conserver la qualité requise, dimensionner plus largement les canalisations secondaires.

Le calcul des débits admissibles $M_{1}$ et $M_{2}$ s'effectuera ainsi qu'il vient d'être indiqué, grâce aux deux tableaux $T_{1}$ et $T_{2}$; il suffira, pour être à même d'établir $T_{2}$, de supposer que toutes les canalisations secondaires sont susceptibles de débiter autant qu'il peut leur être demandé. Cette hypothèse - qui n'intervient que pour des débits supérieurs à $M_{1}$ sur l'une des canalisations secondaires - n'introduit aucune erreur appréciable quand la qualité de fonctionnement recherchée n'est pas trop mauvaise; elle simplifie d'ailleurs beaucoup le calcul et on vérifie en général qu'il est inutile de procéder à des approximations successives théoriquement possibles.

Bien entendu, il suffira, dans la plupart des cas, de se limiter aux premiers termes; un ou deux essais, effectués en se fixant des valeurs plausibles, conduiront aux quantités $M_{1}$ et $M_{2}$ les plus convenables pour assurer aux appareils la qualité de fonctionnement recherchée.

\section{QUELQUES CAS CONCRETS DE DÉTERMINATION}

Bien que la méthode exposée soit générale et puisse s'appliquer à toute installation d'alimentation ou de vidange faisant intervenir des appareils fonctionnant indépendamment et de façon intermittente, la plupart des exemples traités se rattachent à des installations sanitaires.

Plusieurs raisons motivent ce choix :
Le but, les appareils et les quantités mises en jeu, ainsi que les difficultés parfois rencontrées dans ces installations sont familiers à chacun.

Le déroulement des calculs est, comme les conclusions générales qu'on peut tirer, identique; les installations sanitaires constituent même des cas plus ardus que ceux présentés, en général, par l'industrie.

Enfin, - et là réside le principal intérêt l'expérience acquise permet de sanctionner la valeur des résultats obtenus.

\section{I. - Colonne d'alimentation de 12 lavabos sem- blables.}

Supposons que ces lavabos constituent les seuls appareils sanitaires d'un hôtel meublé modeste (fig. 4). De l'avis de personnes autorisées, nous prendrons pour:

- période de pointe : $T=2 \mathrm{~h}$

(de 8 à 10 heures du matin);

- débit unitaire: $Q=0,1 \mathrm{l} / \mathrm{sec}$.

(normes françaises);

- volume moyen nécessaire : $V=201$.

(une personne par chambre).

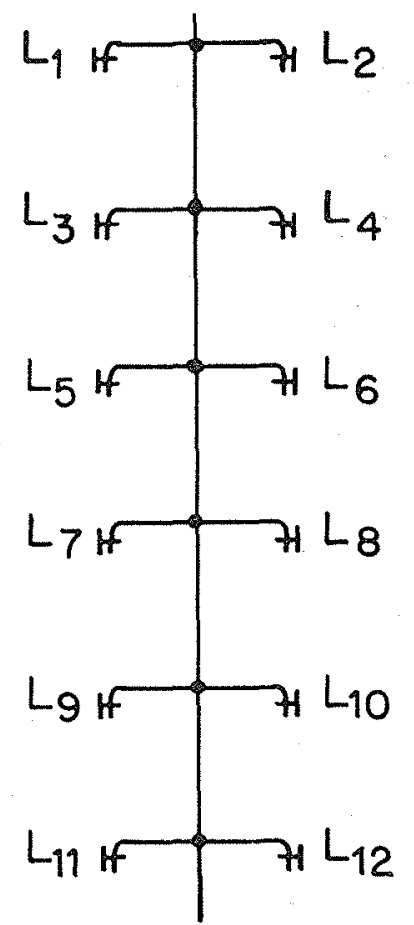

fig. 4 
II s'ensuit, d'après nos précédentes notations:

$$
\begin{aligned}
& d=\frac{20}{0,1}=200 \\
& p=\frac{200}{2 \times 3.600}=\frac{1}{36} \\
& q=\frac{35}{36}
\end{aligned}
$$

11 reste à déterminer la qualité de fonctionnement; admettons que les utilisateurs aient lieu d'être satisfaits, lorsque le débit obtenu n'est pas, en moyenne, inférieur au débit nominal supposé demandé plus de 5 fois sur 100. Cela revient à poser : $m=0,95$.

Calcul de la probabilité pour que 11 parmi les 12 lavabos considérés demandent un débit:

$$
\begin{aligned}
& \text { Nul : } P_{0}=q^{11} \ldots \ldots \ldots=0,733 \\
& O^{1}, 1: P^{\prime}=c_{11} \text { p.q. }{ }^{10} \ldots=\underline{0,230} \\
& \text { inférieur à } O^{0}, 1 \ldots \ldots \ldots=0,963
\end{aligned}
$$

Le débit admissible est donc $2 \mathrm{Q}=0,2 \mathrm{l} / \mathrm{sec}$. conférant à l'installation une qualité meilleure que 0,95 .

Cette qualité sera effectivement atteinte:

en dimensionnant l'installation pour assurer un débit de $\mathrm{O}^{1}, 1$ sur les deux appareils supérieurs $L_{1}$ et $L_{2}$, en limitant le débit possible de chaque lavabo à sa valeur nominale $0^{\prime}, 1 / \mathrm{sec}$, question sur laquelle nous allons revenir.

Remarquons sur cet exemple l'intérêt qu'on aura à adopter un débit juste convenable; un dimensionnement pour $0^{\prime}, 3 / \mathrm{sec}$. n'augmente la qualité que de 0.03 ; l'amélioration apportée par le choix d'une valeur supérieure est tout à fait illusoire.

Notons encore que si tous les appareils étaient disposés dans un même plan horizontal et soumis sensiblement aux mêmes pertes de charge, le débit se partagerait également entre tous les appareils en activité à un instant donné. Dans le cas considéré, et avec un débit de $0^{1}, 2 / \mathrm{sec}$. pour la canalisation d'alimentation, un débit de 0,2 3

- $1 / \mathrm{sec}$. serait assuré encore pendant $3 \%$ du temps sur tous les appareils, ce qui permettrait éventuellement de réduire le coefficient de qualité $\mathbf{m}$.

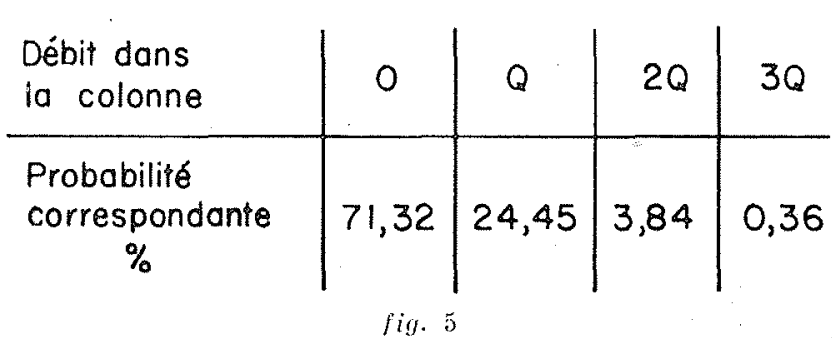

Le regime de la colonne, donné dans ce cas à titre d'information, est fourni par le tableau (5). Le débit le plus probable est un débit nul.

\section{Cas d'appareils dissemblables.}

En vue d'examiner l'influence sur le dimensionnement de l'installation, du fait que les appareils les plus favorisés peuvent fournir un débit supérieur au débit nominal, nous supposerons que les 4 lavabos inférieurs, alimentés par la précédente colonne, ont un débit égal à $0,2 \mathrm{l} / \mathrm{sec}$. Nous conserverons par ailleurs toutes les autres données, ce qui conduit à :

\begin{tabular}{|c|c|c|}
\hline $\begin{array}{c}\text { Type d'appareil } \\
\text { Débit nominal } \\
\text { I/sec. }\end{array}$ & $A_{1}$ & $A_{2}$ \\
\hline$P$ & 0,1 & 0,2 \\
\hline$q$ & $\frac{1}{36}$ & $\frac{1}{72}$ \\
\hline 36 & $\frac{71}{72}$ \\
\hline
\end{tabular}

Suivant la méthode indiquée, nous évaluerons les probabilités de débits croissants demandés à la canalisation en nous limitant, par exemple, à un débit de $0,2 \mathrm{l} / \mathrm{sec}$., quitte à devoir y revenir si les qualités trouvées sont insuffisantes.

\section{Probabilité d'un débit :}

- Nul: $P_{0}=q_{1}^{8} q_{2}^{4}=0,755$

$-0,1 \mathrm{l} / \mathrm{sec}: \mathrm{P}_{1}=\mathrm{c}_{8}^{1} \mathrm{p}_{1} \cdot \mathrm{q}_{1}^{7} \cdot \mathrm{q}_{2}^{4}$

$$
\frac{p_{1}}{q_{1}} P_{0}=0,172
$$

- $0,2 \mathrm{l} / \mathrm{sec}$ : par fonctionnement $1 \mathrm{~A}_{2}$ : $c_{4}^{1} q_{1}^{8} p_{2} \quad q_{2}^{3}=0,042$ par fonctionnement $2 A_{1}$ : $c_{8}^{2} p_{1}^{2} q_{1}^{5}, q_{2}^{4}=0,017$ 


\begin{tabular}{|c|c|c|c|c|c|c|c|}
\hline \multirow{2}{*}{$\begin{array}{l}\text { Débit dans } \\
\text { la colonne } \\
\begin{array}{l}\text { Appareils en } \\
\text { fonctionnement }\end{array}\end{array}$} & \multirow[t]{2}{*}{0} & \multirow{2}{*}{$\frac{\mathrm{Q}}{\mathrm{A}_{1}}$} & \multicolumn{2}{|c|}{$2 Q$} & \multicolumn{2}{|c|}{$3 Q$} & \\
\hline & & & $A_{2}$ & $2 A_{1}$ & $A_{1}+A_{2}$ & $3 A_{1}$ & \\
\hline $\begin{array}{c}\text { Probabilité } \\
\text { correspondante } \\
\qquad \%\end{array}$ & 75,49 & 17,25 & 4,25 & 1,72 & 0,1 & 0,1 & \\
\hline $\begin{array}{l}\text { Propabilité } \\
\text { pour débit } \leqslant \\
\%\end{array}$ & 75,49 & 92,74 & \multicolumn{2}{|c|}{98,71} & \multicolumn{2}{|c|}{98,91} & \\
\hline $\begin{array}{l}\text { Débił M } \\
\text { essayé }\end{array}$ & & & & & \multicolumn{2}{|c|}{$M=3 Q$} & \\
\hline $\begin{array}{c}\text { Qualite sur } \\
A_{1} \\
\end{array}$ & 75,49 & $\frac{7}{8} \cdot 17,25$ & 4,25 & $\frac{6}{8}, 1,72$ & \multicolumn{2}{|c|}{$M-Q$} & $96,1 \times \frac{36}{35}=98,9$ \\
\hline $\begin{array}{c}\text { Qualite sur } \\
\mathrm{A}_{2}\end{array}$ & 75,49 & 17,25 & & & \multicolumn{2}{|c|}{$M-2 Q$} & $92,7 \times \frac{72}{71}=94$ \\
\hline
\end{tabular}

Les résultats, qu'en pratique nous établirons directement sur le tableau (6), nous permettrons de calculer les sommes $\sigma_{1}$ et $\sigma_{2}$ et les qualités correspondantes $\mathrm{m}_{1}=\frac{\sigma_{1}}{\mathrm{q}_{1}}, \mathrm{~m}_{2}=\frac{\sigma_{2}}{\mathrm{q}_{2}}$ relatives au débit $M=0,3 \mathrm{l} / \mathrm{sec}$. essayé. Comme l'installation comporte des appareils semblables, il faudra introduire les facteurs correctifs convenables: ici, $\frac{6}{8}$ et $\frac{7}{8}$ dans la détermination de $\sigma_{1}$

Malgré une augmentation de $50 \%$ par rapport au débit admis pour le cas précédent, la qualité est à peine suffisante sur les appareils $A_{2}$ à fort débit, bien que les utilisateurs ne demandent, suivant nos hypothèses, que le même volume d'eau. Nous voyons, sur cet exemple, l'intérêt qu'il y a à limiter, grâce à des dispositifs convenables, le débit sur tous les appareils servant à des fins analogues, à la même valeur minimum convenable.

Pratiquement, les installations qui ont un fonctionnement correct répondent aux débits calculés ci-dessus :

$0,2 \mathrm{l} / \mathrm{sec}$. est une valeur un peu faible,

$0,3 \mathrm{l} / \mathrm{sec}$. est un dimensionnement assez large.

\section{II. - Alimentation de 14 robinets de chasse} (fig. 7).

Le débit exigé par chaque robinet est par exemple de $1,5 \mathrm{l} / \mathrm{sec}$; nous supposerons que, dans l'immeuble considéré, la période de pointe

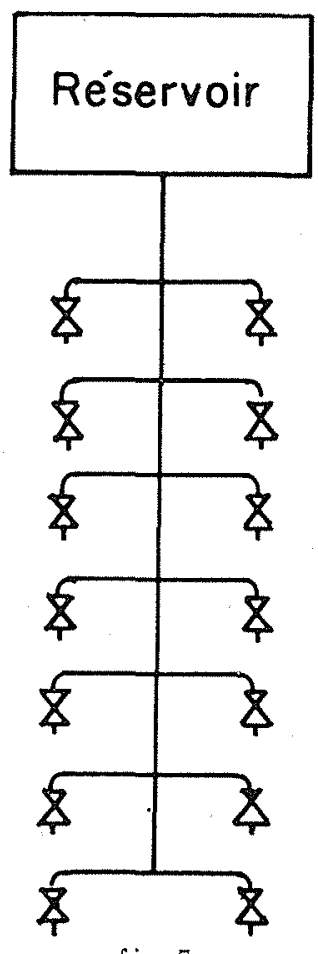

fig. 7 
s'étend sur 2 heures dans la matinée et que le volume moyen nécessaire soit de 40 litres (pour 6 usages par appareil). La qualité que nous voudrons excellente sera fixée à $m: 0,98$.

Nous aurons alors :

$$
\begin{aligned}
& T: 7.200 ; \quad d: \frac{40}{1,5} ; \\
& p: \frac{1}{270} ; \quad q: \frac{269}{270}
\end{aligned}
$$

En faisant abstraction d'un robinet, les probabilités sont les suivantes:

Débit nul : $\mathrm{q}^{13} \ldots \ldots \ldots=0,953$

Débit $1,5 \mathrm{l} / \mathrm{sec}$ : : $C_{13}^{13}$ p.q ${ }^{12}=0,046$

$$
\text { Débit inférieur à } 1,5 \mathrm{l} / \mathrm{sec} .=0,999
$$

Un débit de $3 \mathrm{l} / \mathrm{sec}$. seulement assurera donc un fonctionnement pratiquement parfait; nous voyons ici qu'un calcul d'une simplicité extrême permet d'affirmer l'inutilité d'un dimensionnement plus large. Pourtant, les installations existantes sont dans ce cas plus largement calculées.

\section{1. - Cas de 72 bacs disposés en 12 travées identiques.}

Une telle installation se rencontrera par exemple dans un atelier de décapage; l'eau chaude étant amenée par une canalisation principale (c) et lalimentation de chaque travée étant réalisée par une canalisation secondaire (s) (fig. 8). Si les bacs ont une contenance voisine de 200 litres et sont entièrement remplis en moyenne une fois par heure de travail, à des ins.

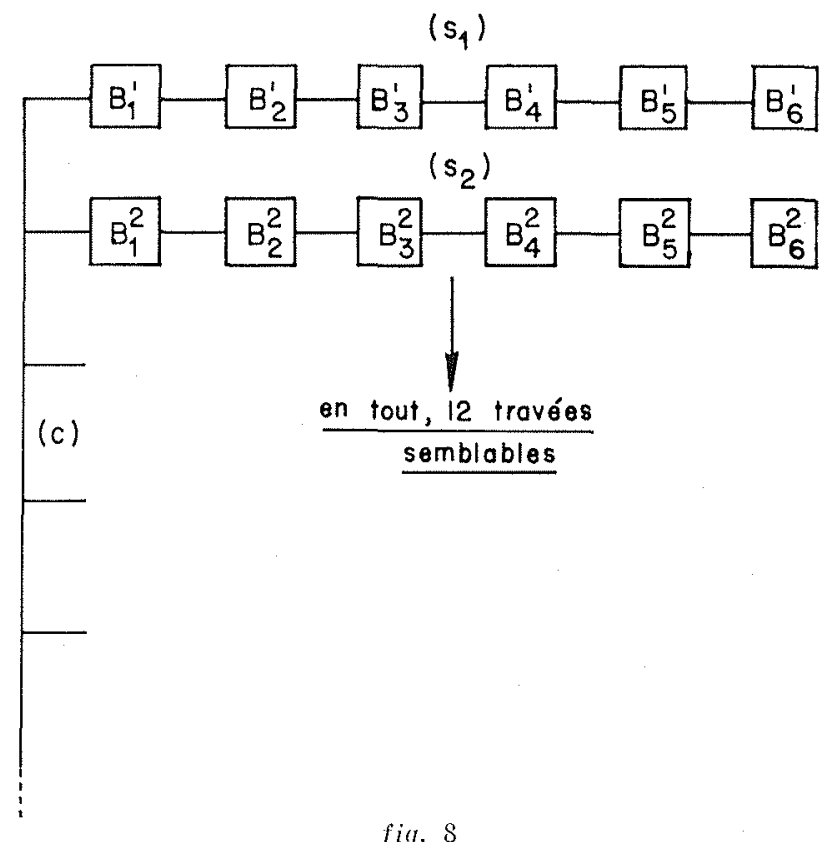

tants n'ayant entre eux aucune relation, nous trouvons pour un débit nominal par bac de $Q=0,55 \mathrm{l} / \mathrm{sec}$ :

$$
p: \frac{1}{10} ; q: \frac{9}{10}
$$

Nous supposerons que le rendement de l'atelier ne sera pas diminué si la qualité est voisine de $m: 0,95$.

Pour déterminer les débits admissibles $M_{1}$ et $M_{3}$ nous allons dresser, ainsi que nous l'avons vu, les deux tableaux $T_{\text {, }}$ et $T_{2}$ (fig. 9), très commodément ici, puisque tous les appareils sont semblables:

$T_{1}$ : en admettant momentanément que le col-

\begin{tabular}{l|c|c|c|c} 
Débit pour 5 bacs & 0 & $Q$ & $2 Q$ & $3 Q$ \\
\hline Probabilité $\%$ & 59,05 & 32,80 & 7,30 & 0,80 \\
\hline Débit admissible correspondant & $Q$ & $2 Q$ & 30 & $4 Q$ \\
\hline Qualité partielle \% & 59,05 & 91,85 & 99,15 & 99,95
\end{tabular}

\begin{tabular}{l|c|c|c|c|c|c|c|c|c|c|c|c} 
Débit pour 66 bacs & 0 & $\mathrm{Q}$ & $2 \mathrm{Q}$ & $3 \mathrm{Q}$ & $4 \mathrm{Q}$ & $5 \mathrm{Q}$ & $6 \mathrm{Q}$ & $7 \mathrm{Q}$ & $8 \mathrm{Q}$ & $9 \mathrm{Q}$ & $10 \mathrm{Q}$ & $11 \mathrm{Q}$ \\
\hline Probabilité $\%$ & 0,1 & 0,7 & 2,5 & 6 & 10,5 & 14,45 & 16,3 & 15,55 & 12,75 & 9,1 & 5,8 & 3,3 \\
\hline Qualité partielle \% & 0,1 & 0,8 & 3,3 & 9,3 & 19,8 & 34,25 & 50,55 & 66,10 & 78,85 & 87,95 & 93,75 & 97,05 \\
\hline
\end{tabular}


lecteur est dimensionné pour le débit maximum qui peut lui être demandé, nous sommes ramenés à un problème déjà traité: calcul, pour 5 bacs, de la probabilité d'un débit nul, égal à $Q$, puis à $2 Q$, en nous limitant à cette valeur qui correspond à $M: 3 \mathrm{Q}=1,65 \mathrm{l} / \mathrm{sec}$, avec une qualité partielle 0,99 , la première rencontrée suffisamment bonne.

$T_{2}$ : le second tableau sera établi en cherchant par un calcul analogue au précédent la probabilité des débits que peuvent demander tous les bacs - à l'exclusion de ceux d'une travée - au cas où les canalisations secondaires seraient dimensionnées pour $6 \mathrm{Q}$ chacune. Nous vérifions ici que cette hypothèse, qui simplifie considérablement le calcul, est légitime, car l'erreur qui s'introduit ainsi correspond aux configurations où plus de 3 bacs débitent à la fois dans la même travée; or:

- d'une part, ces configurations sont relativement peu nombreuses pour des débits inférieurs ou égaux à $M_{2}$,

- d'autre part, la probabilité d'un débit égal ou supérieur à 3 dans une travée est de l'ordre de $1 . \%$.

Dans le cas envisagé, la somme des probabilités rangées dans $T_{2}$ ne dépasse 0,95 que lorsque nous considérons des débits allant jusqu'à $11 \mathrm{Q}$

Le calcul se fait assez vite en s'aidant des logarithmes pour quelques valeurs espacées du débit et en déterminant les valeurs intermédiaires des probabilités en passant de l'une à la voisine, grâce à la règle à calcul.

Nous essaierons donc $M_{2}: 12 Q$; le débit nominal sur un appareil quelconque sera obtenu dans les cas suivants :
Il faut mentionner que des hypothèses supplémentaires pourraient être introduites, tenant compte par exemple du nombre maximum possible d'appareils en fonctionnement lié au nombre des ouvriers de l'usine, mais, si l'hypothèse des puisages sans relation entre eux est sauvegardée, le précédent calcul donne pratiquement un résultat suffisant

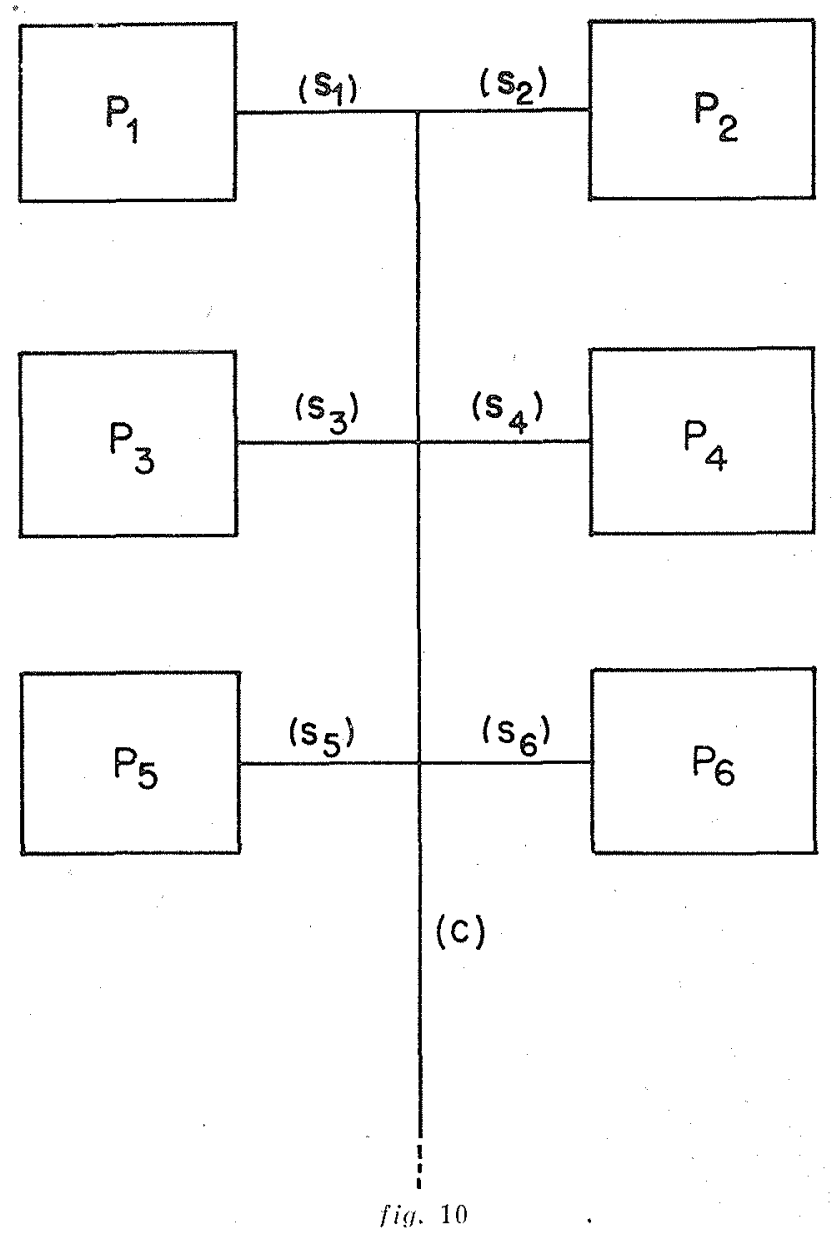

\begin{tabular}{|l|c|c|c|}
\hline Débit dans $(\mathrm{s})=$ & $\mathrm{Q}$ & $2 \mathrm{Q}$ & $3 \mathrm{Q}$ \\
\hline Débit correspondant dans $(\mathrm{c}) \leqslant$ & $11 \mathrm{Q}$ & $10 \mathrm{Q}$ & $9 \mathrm{Q}$ \\
\hline Probabilité : & $0,59 \times 0,975$ & $0,328 \times 0,937$ & $0,07 \times 0,88$ \\
\hline
\end{tabular}

Soit, en faisant la somme, une qualité m : 0,946 ; peu différente de celle que nous nous étions fixée; nous retiendrons donc, pour le dimensionnement, les valeurs:

$$
M_{1}=3 Q ; \quad M_{2}=12 Q
$$

soit respectivement, $50 \%$ et $16 \%$ des débits maxima dans (s) et (c), le dernier pourcentage pouvant sembler très faible pour une telle installation.

\section{IV. - Alimentation en eau de 6 pavillons d'ha- bitation.}

Prenons le cas d'un émetteur de radiodiffusion - ou d'une sous-station électrique - assez éloignée de tout centre d'habitation pour nécessiter la construction aux alentours de 6 pavillons pour le personnel de surveillance (fig. 10). Chaque pavillon sera prévu pour loger, par exemple, 


\begin{tabular}{c|c|c|c|c|c} 
Appareils & $\begin{array}{c}A_{1} \\
W_{-}\end{array}$ & $\begin{array}{c}A_{2} \\
\text { Lavabo }\end{array}$ & $\begin{array}{c}A_{3} \\
\text { Bidet }\end{array}$ & $\begin{array}{c}A_{4} \\
\text { Evier }\end{array}$ & $\begin{array}{c}A_{5} \\
\text { Douche }\end{array}$ \\
\hline Débits (I/sec) & 0,1 & 0,1 & 0,1 & 0,2 & 0,25 \\
\hline $\begin{array}{c}\text { Volumes moyens } \\
\text { Nécessoires (1) }\end{array}$ & 40 & 25 & 15 & 10 & 50 \\
\hline$p \%$ & 5,5 & 3,5 & 2,1 & 0,7 & 2,8 \\
\hline$q \%$ & 94,5 & 96,5 & 97,9 & 99,3 & 97,2 \\
\hline \multicolumn{5}{c}{ fig. 11}
\end{tabular}

4 personnes; le tableau (11) donne pendant la période de pointe générale - deux heures dans la matinée - les débits, les volumes nécessaires et les probabilités de fonctionnement de chacun des appareils sanitaires.

Si nous recherchons une qualité $M$ voisine de 0,94 , le tableau $T$. (fig. 12) nous fixe, comme valeur minimum possible pour $\mathrm{M}_{1}: 0,35 \mathrm{l} / \mathrm{sec}$.

L'essai de $M_{2}: 0,60 \mathrm{l} / \mathrm{sec}$. conduit, grâce aux valeurs fournies par $T_{2}$, aux qualités 0,94 et 0,95 respectivement pour l'évier et la douche et à des qualités supérieures pour les autres appareils.

Le dimensionnement de l'installation pourra donc être basé sur:

$$
M_{1}: 0,35 \mathrm{l} / \mathrm{sec} ; \quad M_{2}: 0,60 \mathrm{l} / \mathrm{sec} \text {. }
$$

Notons à ce propos que $M_{2}$ n'est pas forcément, dans ce cas, un multiple de $M_{1}$.

\begin{tabular}{|c|c|c|c|c|c|c|c|c|c|c|c|}
\hline \multirow{2}{*}{$\begin{array}{l}\text { Débit dans la } \\
\text { canalisation } \\
\text { Appareils en } \\
\text { fonctionnement }\end{array}$} & \multirow[t]{2}{*}{0} & \multicolumn{3}{|c|}{0,9} & \multicolumn{4}{|c|}{0,2} & 0,25 & & \\
\hline & & $A_{1}$ & $A_{2}$ & $A_{3}$ & $A_{4}$ & $A_{1}+A_{2}$ & $A_{1}+A_{3}$ & $A_{2}+A_{3}$ & $A_{5}$ & & \\
\hline $\begin{array}{l}\text { Probabilité } \\
\text { correspondante \% }\end{array}$ & 86,15 & 5,05 & 3,15 & 1,85 & 0,6 & 0,15 & 0,1 & 0,05 & 2,5 & & \\
\hline $\begin{array}{l}\text { Debit M } \\
\text { essayé }\end{array}$ & & & & & & & & & & $M_{4}=0$ & 35 \\
\hline $\begin{array}{c}\text { Qualité sur } \\
A_{1}\end{array}$ & 86,15 & & 3,15 & 1,85 & 0,6 & & & 0,05 & $\cdots$ & & \\
\hline $\begin{array}{c}\text { Qualité sur } \\
\mathrm{A}_{2}\end{array}$ & 86,15 & 5,05 & & 1,85 & 0,6 & & 0,4 & & & & \\
\hline $\begin{array}{c}\text { Qualité sur } \\
A_{3}\end{array}$ & 86,15 & 5,05 & 3,15 & & 0,6 & 0,15 & & & & & \\
\hline $\begin{array}{l}\text { Qualité sur } \\
A_{4}\end{array}$ & 86,15 & 5,05 & 3,15 & 1,85 & - & & & & & $M_{i}-0,2$ & $96,2 \cdot \frac{100}{99,3}=96,85 \%$ \\
\hline $\begin{array}{c}\text { Qualité sur } \\
\mathrm{A}_{5}\end{array}$ & 86,15 & 5,05 & 3,15 & 1,85 & & & & & & $M_{1}-0,25$ & $96,2 \cdot \frac{100}{97,2} 98,8 \%$ \\
\hline
\end{tabular}

fist. 12

Le régime de fonctionnement d'une canalisation secondaire est:

\begin{tabular}{c|c|c|c|c|c|c|c|c|}
$\begin{array}{c}\text { Débit dans } \\
\text { la canalisation }\end{array}$ & 0 & 0,1 & 0,2 & 0,25 & 0,3 & 0,35 & \\
\hline Probabilité \% & 86,15 & 10,05 & 0,90 & 2,50 & 0,05 & $0,35 \%$
\end{tabular}

En examinant la probabilité des débits croissants demandés par l'ensemble de 5 canalisations semolables, on obtient :

\begin{tabular}{|c|c|c|c|c|c|c|c|}
\hline Débit demandé. & 0 & 0,1 & 0,2 & 0,25 & 0,3 & 0,35 & 0,4 \\
\hline Probabilité correspondante $\%$... & 47,45 & 27,70 & 8,95 & 6,9 & 2,0 & 4,15 & 0,35 \\
\hline Probabilité pour un débit $\leqslant \%$ & 47,45 & 75,15 & 84,10 & 91 & 93 & 97,15 & 97,50 \\
\hline
\end{tabular}


Essai de $M_{2}=0,60 \mathrm{I} . / \mathrm{sec}$. pour le collecteur :

Fonctionnement correct sur $A_{5}$ si :

$$
\begin{array}{ll}
\mathrm{S}=0, \quad c \leqslant 0,6-0,25=0,35: & \text { Probabilité }: 0,886 \times 0,9715=0,861 \\
\mathrm{~S}=0,1, c \leqslant 0,6-0,35=0,25: & \text { Probabilité }: 0,103 \times 0,91=0,093
\end{array}
$$

Qualité sur $\mathbf{A}_{5}$

Probabilité : $0,103 \times 0,91=0,093$

Fonctionnement correct sur $A_{4}$ si :

$$
\begin{array}{ll}
\mathrm{S}=0, \quad c \leqslant 0,6-0,2=0,4: & \text { Probabilité }: 0,868 \times 0,975=0,847 \\
\mathrm{~S}=0,1, c \leqslant 0,6-0,3=0,3: & \text { Probabilité }: 0,101 \times 0,93=0,094
\end{array}
$$

Qualité sur $\mathbf{A}_{4}$

$\begin{array}{ll}\text { Probabilité : } & 0,868 \times 0,975=0,847 \\ \text { Probabilité : } & 0,101 \times 0,93=0,094\end{array}$

* La probabilité du débit nul considéré ne fait pas intervenir l'appareil en étude $A_{i j}$ et :

$$
\left.0,886=\frac{0,8615}{q_{5}}=\frac{0,8615}{0,972}\right]
$$

Le classement des appareils par ordre de qualité se modifie suivant les valeurs données à $M_{1}$ et $M_{2}$; même en gardant ici $M_{1}: 0,35 \mathrm{l} / \mathrm{sec}$., l'adoption de $M_{2}: 0,55 \mathrm{l} / \mathrm{sec}$. ferait que l'appareil le plus défavorisé ne serait plus l'évier, mais la douche, dont la qualité tombe alors à la valeur 0,91 inacceptable.

Nous pensons que ces quelques exemples auront prouvé la validité des hypothèses faites, ainsi que la relative simplicité de la méthode indiquée; nous espérons que ce procédé de calcul pourra guider utilement dans la détermination des débits admissibles optima, détermination qui semble avoir reposé jusqu'ici, en général, sur des bases empiriques assez incertaines.
Puisque nous avons traité de cas d'installations sanitaires, mentionnons encore, dans ce domaine, l'intérêt d'une introduction de la notion de qualité qui supprimerait les difficultés provenant de fonctionnements insuffisants dont se plaignent les utilisateurs; les installations pourraient, en effet, être prévues poụr des débits et des qualités "raisonnables» — au besoin codifiés par catégories - une insuffisance en un point n'étant, dès lors, à craindre que par usage abusif commis par ailleurs:

Avant de terminer, nous tenons à remercier M. L. BOURCIER, Ingénieur-Conseil, qui a attiré notre attention sur le présent problème et nous a fourni l'essentiel des données numériques utilisées dans les applications pratiques. 